\title{
EL MODELO DEL SISTEMA VIABLE: UNA REFERENCIA ESTRATÉGICA PARA EL ESTUDIO ORGANIZACIONAL DEL SECTOR COOPERATIVO CHILENO
}

\author{
POR \\ Pedro Antonio NARVARTE ARREGUI y \\ Catalina CAREAGA DÍAZ ${ }^{1}$
}

\section{RESUMEN}

El Sector Cooperativo Chileno (SCCh) puede ser explicado como un sistema de actividad humana de alta complejidad, el cual persigue mantener una existencia autónoma, es decir, ser viable. Desde esta perspectiva, el Modelo del Sistema Viable (MSV), como referencia conceptual, presenta una oportunidad real para estudiar la organización sectorial del cooperativismo en Chile.

El objetivo central del trabajo, se refiere a estudiar la viabilidad del SCCh en un contexto de organización sectorial, considerando el entramado social, legal y económico del país en la actualidad.

Para ello, soportado por una metodología sistémica, se realizaron: una caracterización de la situación problema del sector -identificando algunos factores relevantes en los ámbitos de estructura de mercado, regulaciones legales, e inter cooperación-, un diagnóstico organizacional y la propuesta de un conjunto de orientaciones para su desarrollo.

De lo anterior se concluye que existe una pertinencia entre las características del caso de estudio y el enfoque teórico metodológico. La metodología, toma herramientas probadas en otras prácticas organizacionales, tal como VIPLAN, y las aplica al SCCh. Su aporte en el campo de estudio se orienta en torno a una mirada holística de la organización y la promoción de su viabilidad, generando de este modo un enfoque que entrega estrategias concretas de

\footnotetext{
${ }^{1}$ Integran el Centro para la Integración Ingeniería y Sociedad (CIIS) de la Facultad de Ingeniería de la Universidad de Santiago de Chile (USACH). Direcciones de correo electrónico: pedro.narvarte@usach.cl y catalina.careaga@usach.cl.
}

REVESCO No 121 - Segundo Cuatrimestre 2016 - ISSN: 1885-8031 - www.ucm.es/info/revesco

http://dx.doi.org/10.5209/rev_REVE.2016.v121.49702

Fecha de recepción: 30/06/2014

Fecha de aceptación: 24/07/2015 
desarrollo sectorial, superando la aproximación de caracterización descriptiva. Así, entregamos un modelo diagnóstico del Sector Cooperativo Chileno y proponemos lineamientos que apoyen su desarrollo organizacional.

Palabras Clave: Enfoque sistémico, viabilidad organizacional, cibernética organizacional, cooperativismo, estrategias de desarrollo sectorial.

Claves ECONLIT: D790, M130, M190, P130.

\title{
EL MODELO DEL SISTEMA VIABLE: UNA REFERENCIA ESTRATÉGICA PARA EL ESTUDIO ORGANIZACIONAL DEL SECTOR COOPERATIVO CHILENO
}

\begin{abstract}
The Chilean Cooperative Sector (SCCh) can be explained as a human activity system of high complexity, which seeks to maintain an independent existence, in example, be viable. From this perspective, the Viable System Model (MSV) as conceptual reference presents a real opportunity to study the organization of the cooperative sector in Chile.
\end{abstract}

The central objective of the work refers to study the feasibility of SCCh in a context of sectorial organization, considering the social, legal and economic fabric of the country today.

To do this, supported by a systemic methodology were performed: a characterization of the problem situation of the sector -identifying some relevant factors in the areas of market structure, legal regulations and inter cooperation-an organizational diagnosis and proposed a set guidelines for its development.

From the above it is concluded that there is relevance between the characteristics of the case study theoretical and methodological approach. The methodology takes tested in other organizational practices such as VIPLAN tools, and applies the SCCh. Its contribution in the field of study is oriented around a holistic view of the organization and promotion of their viability, thereby generating an approach that delivers specific sectorial development strategies, surpassing the approximation of descriptive characterization. Thus, we provide a diagnostic model of the Chilean Cooperative Sector and propose guidelines to support their organizational development.

Keywords: Systemic approach, organizational viability, organizational cybernetics, 
cooperativism, sectorial development strategies.

\section{INTRODUCCIÓN}

El desarrollo histórico del cooperativismo en Chile ha sido fluctuante e inestable en el tiempo. Sus inicios se remontan a 1887, con la formación de la Cooperativa de Consumo Esmeralda en la ciudad de Valparaíso (Del Campo y Radrigán, 1998). Luego de esta primera práctica conocida, la existencia de experiencias cooperativas de variada naturaleza ha sido una constante en el país. Desde 1924, año en que se promulga la primera ley sobre cooperativas, se observa una progresiva presencia del Estado y un crecimiento lento pero sostenido del SCCh. Sin embargo, es en la década del "60 cuando éste advierte un ingente crecimiento, potenciado por la reforma agraria y las políticas generales de promoción popular de la época.

A inicios de los '70, el movimiento queda atrapado en el proceso de polarización social y política del gobierno de Allende sumergiéndose en una indiferencia gubernamental, la que se convierte en vigilancia durante el régimen militar (Del Campo y Radrigán, 1998). La ideología neoliberal (López Garay, 1997) legitimó al libre-mercado como único mecanismo de coordinación social, relegando al Estado a un rol subsidiario. En este contexto las prácticas cooperativas no sólo fueron percibidas con sospecha, sino que se tornaron sectorialmente debilitadas.

El regreso a la democracia, desde 1990 en adelante, engendró en el mundo cooperativo mayores esperanzas de las que ha comprometido y concretado a la fecha la política pública. Es así como durante las últimas tres décadas el cooperativismo chileno sigue manteniendo un bajo perfil en el contexto económico nacional, lo cual, a nuestro juicio, da cuenta de otro fenómeno más complejo relacionado con la renuncia ideológica al modelo de empresa cooperativa como una alternativa válida para el desarrollo económico, noción predominante no sólo en el sector empresarial privado, sino también al interior de la institucionalidad pública.

Según el Directorio de Cooperativas del DECOOP (http://www.decoop.cl), actualmente el universo cooperativo acumulado está compuesto por 5006 empresas, de las cuales 1.325 son calificadas como activas por el Ministerio de Economía -en adelante MINECON- por haber presentado antecedentes de movimiento durante los últimos cinco años. Las cooperativas activas, que se desenvuelven en distintos ámbitos de la economía, 
agrupan hoy a 1.697.972 asociados y manejan un volumen de activos de MM\$2.586.973 y un Patrimonio de MM\$ 1.122.206 (DECOOP, 2014).

Basado en los antecedentes expuestos, establecimos como hipótesis central de la Investigación el siguiente postulado: "el aprendizaje, producto del estudio de la empresa cooperativa y la intercooperación, modeladas como un sistema viable, ayudarán a orientar y dirigir la investigación diagnóstica del fenómeno cooperativo en Chile, con el propósito de establecer estrategias para el estudio, educación y la práctica cooperativa en el contexto económico, social y legal actual de este país".

En este contexto formulamos como objetivo central: "estudiar la viabilidad del SCCh en un contexto de organización sectorial, considerando el entramado social, legal y económico del país en la actualidad". Esto se llevó a cabo apoyado por un marco metodológico y conceptual situado en la sistémica y la cibernética organizacional (Narvarte, 2002), en el cual el Modelo de Sistema Viable (Beer, 1988; Narvarte, 2002; Espejo y Reyes, 2011) cumple un papel fundamental para estudiar y conducir cambios organizacionales.

\section{APROXIMACIÓN METODOLÓGICA}

\subsection{Metodología}

La aproximación metodológica propuesta, ilustrada en la Figura 1, sigue claramente el patrón establecido en la forma básica de la Metodología de Sistemas Blandos (Checkland, 1993; Checkland y Scholes, 1994; Checkland y Poulter, 2010), sin embargo, está más focalizada al estudio e intervención en organizaciones complejas, que al estudio de situaciones complejas en las organizaciones. Debido a esto, mostrará sustanciales variaciones respecto a las metodologías -convencional y desarrollada- que plantea Checkland, de modo tal de adecuar su operatoria al contexto de la investigación. 
Figura 1. Metodología MECISIOR

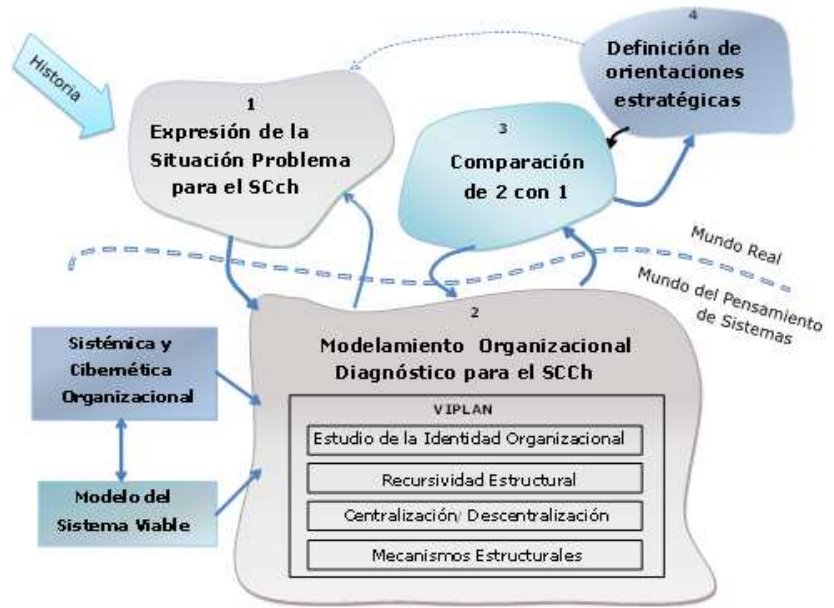

Fuente: Adaptada de NARVARTE, 2002. Figura 2.2, pág. 35

La Figura 1, muestra la metodología que apoyará este trabajo, la que llamaremos "Metodología Sistémico-Cibernética para el Estudio de Fenómenos Organizacionales Complejos (MESICIOR)” (Narvarte, 2002), debidamente adaptada para focalizar solo el modelamiento organizacional. Esta metodología, corresponde a un aporte intelectual orientado a cooperar con los estudiosos y responsables de las organizaciones -cualesquiera sean-, en la comprensión y aprendizaje orientado a intervenirlas efectivamente.

Las actividades desarrolladas para el estudio del SCCh (Figura 1) se agruparon en cuatro etapas de la intervención. Éstas son:

1. Expresión de la situación problema, fase en la cual se describió críticamente la organización sectorial del SCCh y su entorno, basándose en el material primario y secundario recolectado durante la realización del trabajo.

2. Modelamiento organizacional diagnóstico para el $\mathbf{S C C h}$, el que se efectuó siguiendo el método para el estudio de organizaciones VIPLAN (Espejo, 1997; Espejo, Bowling y Hooversadt, 1999; Narvarte, 2002; Espejo y Reyes, 2011; Espejo, 2015) desarrollado durante años en la aplicación del Modelo de Sistema Viable en organizaciones humanas. Contempla los siguientes pasos: (i) Estudio de la identidad organizacional para el SCCh; (ii) Recursividad estructural para el SCCh; (iii) Grado de centralización/ descentralización que caracteriza la regulación del SCCh; (iv) Mecanismos estructurales para la regulación del SCCh (capacidad de adaptación y cohesión organizacional sectorial).

3. Comparación de etapas 2 y 1, donde se llevó a cabo una contrastación entre lo diagnosticado y lo establecido en la situación problema definido en la etapa 1. 
4. Definición de orientaciones estratégicas, donde se perfilaron las orientaciones organizacionales necesarias para dar solución a lo expresado en la situación problema, proponiendo transformaciones consecuentes con lo establecido en la etapa 2. Para que estos cambios se materialicen deberán ser aceptados por la comunidad organizacional y ser factibles de realizar en su dominio cultural.

\subsection{El modelo del sistema viable (MSV)}

El MSV fue creado por Stafford Beer y reúne el trabajo de muchos autores sistémicos, quienes han aportado desde diversos campos disciplinares tales como: la neurofisiología, la cibernética, la neurocibernética, entre otros (Beer, 1985; Narvarte, Castillo y Torres, 2006; Narvarte, 1995, 2002, 2006; Espejo y Reyes, 2011; Espejo, 2015; Medina, 2013). Este modelo representa un modo de entendimiento de las organizaciones humanas que deja atrás los modelos jerárquicos y reduccionistas que caracterizan a los métodos de la administración tradicional. Fue creado con el propósito de entregar una alternativa científica real para el estudio de organizaciones de actividad humana, bajo la premisa de cumplir con la condición de sostener la viabilidad organizacional como único requisito.

El MSV postula una organización recursiva, tal como se ilustra en la Figura 2.

Figura 2. Organización Recursiva

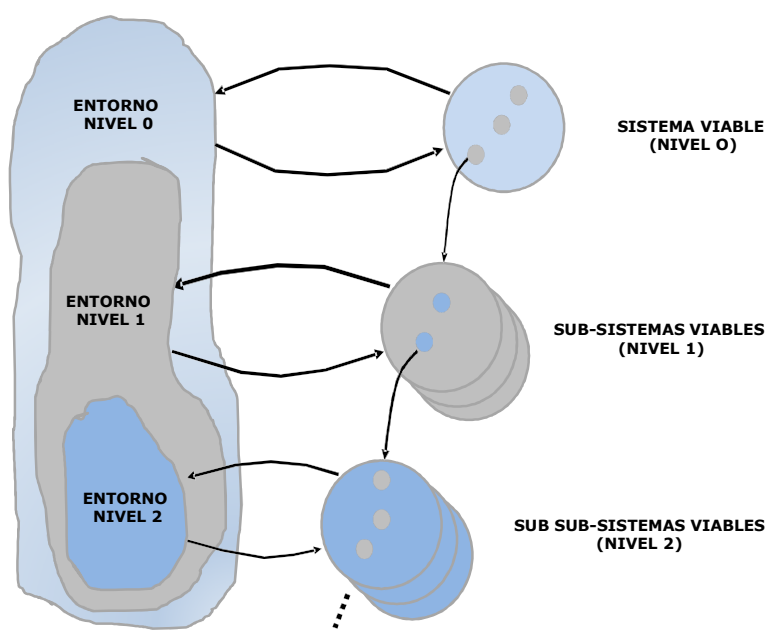

Fuente: Espejo y Reyes, 2011, p 96

Lo anterior significa que los sistemas viables -o autónomos- deben estar en sí compuestos por sistemas viables o autónomos, cada uno preocupado por su propio desarrollo. De esta manera los responsables de la administración, en cada nivel de la organización, 
definen sus políticas a través de las tareas que implementarán en forma autónoma aunque cohesionados estructuralmente.

Para cada actividad viable identificada en el mapa recursivo, existen cinco subsistemas, los que se ilustran en la figura 3.

Figura 3. Modelo del Sistema Viable

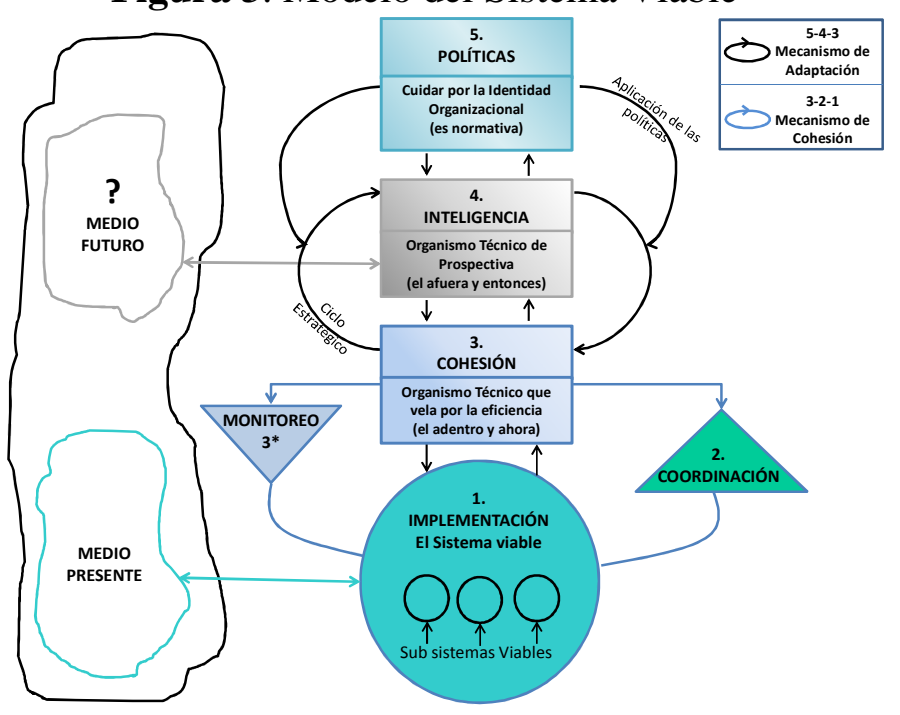

Fuente: Elaboración Propia

Sub-sistema 1.Función implementación.

Corresponde al quehacer de la organización. Se refleja en las actividades primarias (viables), aquellas que constituyen la esencia y razón de existir de la organización, dotándola de sentido y definiendo su identidad. Van acompañadas siempre de actividades de apoyo no autónomas, para su regulación.

Sub-sistema 2.Función de coordinación.

La función de coordinación filtra la variedad recursiva, para que la tarea de quienes ejercen la función de cohesión se alivie. La coordinación es diseñada de modo que permita lenguajes y protocolos de comunicación y conversaciones entre las actividades primarias de un mismo nivel recursivo, a fin de cuidar la consolidación y cohesión del sistema en la ejecución de sus tareas.

\section{Sub-sistema 3.Función de cohesión.}

El interés fundamental de la función de cohesión está en la complejidad organizacional interna, esto es lo que suele llamarse "el adentro y el ahora" (Beer, 1985), del cual debe ser un 
atenuador y habilitador efectivo. Esto es crucial para la constitución del mecanismo de cohesión. Para esto la función de cohesión necesita una apreciación precisa de los logros y capacidades del sistema 1 , así como de la coordinación de potencialidades y requerimientos. En este sentido la función de cohesión es una forma de control que respeta y habilita la autonomía de las actividades viables o primarias en una organización. Incluye dentro de sí un monitoreo esporádico y conocido $\left(3^{*}\right)$, el que asegura la delegación de confianza al siguiente nivel recursivo.

Sub-sistema 4.Función inteligencia.

La función inteligencia tiene su ámbito de acción en el entorno de la organización, preocupándose de su prospectiva y desarrollo futuro (el afuera y el mañana, las oportunidades y amenazas), constituyendo la piedra angular para lograr su adaptación.

\section{Sub-sistema 5.Función política.}

La función política, es normativa y está encargada de dar clausura al sistema viable, cuidando la integridad y la identidad organizacional del sistema.

\section{Mecanismos Reguladores.}

Estas 5 funciones antes presentadas, operan dinámicamente conformando dos mecanismos reguladores: el Mecanismo de Adaptación (funciones 3-4-5) y el Mecanismo de Cohesión (funciones 1-2-3), tal como lo indica la figura 3. El Mecanismo de Adaptación busca la efectividad organizacional -hacer lo correcto en relación a lo que nos depara el futuro-, mientras que el Mecanismo de Cohesión asegura la eficiencia -hacer bien aquello que hago, sin cuestionar el quehacer-. Estos dos mecanismos se complementan sinérgicamente para asegurar la viabilidad organizacional, es decir la capacidad de mantener una existencia separada (Espejo y Reyes, 2011: 92). 


\section{ESTUDIO DE LA ORGANIZACIÓN PARA EL SCCH}

\subsection{Expresión de la situación problema}

Para expresar la situación problema del SCCh se identificaron sus entidades relevantes diferenciadas en dos grandes segmentos, a saber: aquellas que adoptan el papel de vivir los problemas de la realidad cooperativa, que serán reunidos en el Sistema Contenedor del Problema (SCP) y aquellas otras entidades que serán movidas por la vocación de ayuda a resolver dichos problemas, quienes conformarán el Sistema Solucionador del Problema (SSP). (Rodríguez, 1994; Narvarte 2002, Narvarte y Rodes, 2004).

Entre las primeras se cuentan: los socios, las cooperativas de base y las cooperativas del tipo federativo. Imagin-i-zando (Morgan, 1999) la organización cooperativa como un organismo vivo: los socios representan las células; las cooperativas los órganos; las federaciones, organismos públicos de fomento y regulación, sumados a otros entes de intercooperación, son parte de los tejidos nerviosos cuyo rol es permitir la coordinación, regulación e interrelación entre las células y los órganos, así como también entre los órganos del organismo. De esta manera, la viabilidad del sector depende en gran medida de la correcta sincronía entre la estructura que sustente orgánicamente a todos estos componentes.

El otro grupo está conformado por aquellas entidades que muestran vocación para resolver los problemas que sufre el SCCh. Entre ellas se cuentan: los legisladores, el Gobierno, las universidades, los centros de investigación, y aquellos organismos extranjeros relacionados con la economía social. Estas entidades ejercen su acción desde el entorno proponiendo soluciones para el mejoramiento de la operación sistémica. Siguiendo la metáfora anterior, este grupo representa los elementos que desde el ambiente intentan proteger y alivianar las situaciones que el organismo siente como peligrosas, además de contribuir a la visualización por parte del sistema de aquellas oportunidades de desarrollo existentes en el entorno.

\section{- Sintomatología ORGANizACiONAL}

En cuanto a la sintomatología organizacional, decimos que una restricción para el sector cooperativo chileno lo representa la obligación de adaptarse a las condiciones económicas

\footnotetext{
${ }^{2}$ Para profundizar este punto ver: Narvarte, P. y Rodes, D. (2004) Expresión de la Situación Problema para el Sector Cooperativo Chileno: Una Aproximación Sistémica. Revista de Cooperación Internacional de la ACI (Asociación Cooperativa Internacional).Volumen 37 No 1- 2004, p. 7-22.
} 
heredadas del régimen militar, que con ciertos matices se mantienen en lo sustancial hasta hoy. En este contexto, se producen variados conflictos que afectan tanto las relaciones al interior del movimiento, como las de éste con su entorno. Entre los conflictos más relevantes, podemos mencionar:

(a) Conflicto valorativo: valores cooperativos v/s neoliberalismo. La legislación chilena no define un marco valorativo para el movimiento cooperativo chileno, sin embargo sí lo hacen los órganos representantes del movimiento, tales como la Confederación Nacional de Cooperativas de Chile -en adelante CONFECOOP-, la Federación Chilena de Cooperativas de Ahorro y Crédito -en adelante FECRECOOP- y otras entidades que suscriben a la declaración de principios establecida por la Alianza Cooperativa Internacional -en adelante ACI- $^{3}$. En contraposición al sentido de la declaración de la ACI encontramos la visión y actitud individualista del neoliberalismo. En este contexto los valores de solidaridad, participación democrática y cooperación promovidos por el cooperativismo, son deslegitimados por los dispositivos de la economía de libre mercado que operan en la sociedad chilena actual.

(b) Restricciones político-normativas; Las políticas económicas y sociales de las dos últimas décadas han adscrito sustancialmente al modelo neoliberal. En este sentido, se han registrado sólo aislados aportes favorecedores del cooperativismo, entre los cuales se cuentan el nuevo marco normativo (Ley $\mathrm{N}^{\circ}$ 19.832), y el programa de fortalecimiento BID-FOMIN, entre el Banco Interamericano del Desarrollo y el Departamento de Cooperativas del MINECON. En términos concretos, la promulgación de la llamada "nueva Ley de cooperativas" ha permitido flexibilizar una serie de barreras burocráticas que dificultaban la creación y funcionamiento de éstas. De la misma forma, el convenio entre el gobierno de Chile y el BID suscrito en septiembre del 2004 se propuso el fortalecimiento de la regulación y supervisión del SCCh, así como del propio Departamento de Cooperativas. A partir de lo anterior, podemos decir que si bien ha existido voluntad de permitir al cooperativismo chileno adaptarse a los nuevos tiempos, no se observa una preocupación particular por el desarrollo del sector.

(c) El nuevo socio cooperativo; En relación a los nuevos socios; la obligación de búsqueda de nichos de mercados que amplíen su cobertura más allá de la trama social de la cual surgen,

\footnotetext{
${ }^{3}$ La declaración sobre identidad cooperativa de la Alianza Cooperativa Internacional (ACI), establece un acuerdo amplio acerca de: una definición genérica de cooperativa, sus valores y principios (ACI, web: www.ica.coop/ica/es/esprinciples.html).
} 
sumado a la búsqueda utilitaria y comercial de éstas organizaciones promovida por el contexto de competencia mercantil, ha dado origen a un nuevo socio cooperativo. Este "nuevo socio", que se caracteriza por ser un mero cliente que antepone su beneficio personal por sobre la participación y la cooperación, es particularmente visible en las cooperativas de Ahorro y Crédito y de Vivienda Abierta.

(d) La incapacidad de adaptarse al mercado. El modo de transitar desde prácticas empresariales centradas en la satisfacción de cierta comunidad, a otras que permitan con eficiencia y efectividad extenderse a un mercado competitivo, es aún un tema sin resolver para el grueso del movimiento cooperativo chileno.

(e) El individualismo institucional. A raíz de los acontecimientos socio-políticos de las décadas del '70 y '80, cuando las cooperativas vivían el cuestionamiento a sus valores, principios y la pérdida de la ayuda directa del Estado, se genera en ellas una actitud de autodefensa y prácticas individualistas que configuraron un ambiente sectario y competitivo donde muchas veces se negaban los espacios de intercooperación. Estos elementos destruyeron el tejido asociacionista dando origen a lo que llamamos el "individualismo institucional".

\section{- DESAFÍOS DEL SECTOR COOPERATIVO CHILENO}

A la luz de los antecedentes planteados, se puede afirmar que el cooperativismo chileno presenta en la actualidad los siguientes desafíos: (a) Recomponer la identidad cooperativa; (b) Solucionar los problemas estructurales del sector y (c) Dar respuestas a las problemáticas del Chile actual.

El SCCh, ya no puede pretender estar protegido desde las instituciones, sino que debe ser independiente, autosuficiente y autónomo. Además, no puede sobrevivir si se mantiene cerrado al entorno; debe aceptar y asentar su posición en un mundo abierto y globalizado. Eso no significa renunciar a los valores cooperativos, los que adecuadamente adaptados a la época deben guiar su accionar y los que debe sustentar frente a las restricciones que la legislación le impone. Sobre estas bases debe reformularse la "nueva identidad" cooperativa. 


\section{MODELAMIENTO ORGANIZACIONAL PARA EL SCCH}

\subsection{Estudio de la identidad organizacional}

Al estudiar la identidad, se busca determinar lo que la organización es. Esta identidad no es fija ni inmutable, al contrario, se transforma a la par que los miembros de la organización varían su percepción acerca de su razón de ser. A modo de ejemplo de las transformaciones identitarias, decimos que el sector cooperativo chileno no es hoy lo que fue hace 30 años debido a las trasformaciones estructurales que ha vivido el país y sus actores, y en consecuencia nadie podría dar una definición invariante para el Sector.

Para establecer la identidad organizacional, se utilizó la herramienta de "nombrar el sistema", consistente en desentrañar a través de metodologías participativas el significado profundo de la organización. Para ello se determinó: qué hace el $\mathrm{SCCh}$, mediante qué actividades y recursos lo realiza y con qué propósito.

A partir de la información secundaria y de las entrevistas efectuadas, establecimos que la identidad actual del SCCh puede ser referida como sigue:

"Un sistema de estructura asociativa en reconstitución, que reúne a todas las instituciones inscritas bajo la figura jurídica de cooperativas y, por tanto, adscritas a los valores y principios cooperativos, mediante instrumentos legales, asociativos, educativos y de fomento, para permitirles potenciarse, facilitándoles satisfacer sus necesidades de desarrollo y el de las comunidades que atienden, de manera justa, equitativa y solidaria".

Examinando la frase, podemos notar cuatro aspectos destacables: el contexto de reconstitución en el cual se encuentra el cooperativismo chileno; la misión de reunir a las instituciones cooperativas; los medios o instrumentos por los cuales lleva a cabo su quehacer; y por último, el fin que se persigue, que es proveer herramientas para la viabilidad del Sector.

El expresar como constitutivas del sector a las instituciones que tienen la forma jurídica de cooperativas, comporta su adhesión valorativa, que está regida por los principios y valores de la ACI. En este sentido, si bien el comportamiento sectorial no demuestra inconsistencia con tales principios: adhesión voluntaria y abierta, gestión democrática por parte de los socios, participación económica de los socios, sí se advierten debilidades en aquellos relacionados con autonomía e independencia, educación, formación e información, cooperación entre cooperativas e interés por la comunidad. 
En efecto, las cooperativas chilenas en la actualidad tienen limitaciones estructurales que dificultan su autonomía e independencia, herencia -aún no superada- de aquellas prácticas proteccionistas que las instituciones del Estado y/o la Iglesia le ofrecieron antaño. Por otro lado, la educación y la formación cooperativa es débil, la intercooperación se muestra deficiente y el grueso del cooperativismo se encuentra aislado de la sociedad, por lo cual no es visible.

\subsection{Recursividad estructural}

La recursividad estructural está derivada de la identidad del sistema, y permite explicar las actividades fundamentales que lleva -o debiera llevar- a cabo la organización para alcanzar sus propósitos. Estas tareas corresponden a las llamadas actividades primarias, que se encuentran dispuestas en una cascada de sistemas autónomos -o viables- que a su vez están inmersos en sistemas autónomos $-\mathrm{o}$ viables-.

Para lograr una clasificación que dé cuenta de la recursividad estructural del SCCh es necesario adoptar tres criterios: naturaleza sectorial, su relación con el medio y la importancia económica de éstas. Clasificando:

- Según naturaleza sectorial: Cooperativas de producción (agrícolas, campesinas, pesqueras y mineras ), Cooperativas de servicios (consumo, ahorro y crédito, vivienda abierta, vivienda cerrada, veraneo, agua potable, eléctricas, otros servicios: educación, salud, transportes) y Cooperativas de trabajo (las pueden realizar actividades de servicios y/o producción) (DECOOP, 2014).

- Según su relación con el medio: cooperativas cerradas y cooperativas abiertas. Las cooperativas cerradas, están vinculadas con la iniciativa de un grupo social claramente definido -empresa, barrio, parroquia, fuerzas armadas, universidades, etc.-, para satisfacer alguna necesidad específica al colectivo de socios. Ejemplos característicos de cooperativas cerradas son: la totalidad de las cooperativas de consumo en Chile, las cooperativas de vivienda cerradas y las cooperativas de veraneo. Las cooperativas abiertas, se caracterizan por que el grupo social al que sirven es dinámico en el tiempo, no tienen vínculos institucionales ni de ningún tipo que los reúna que no sea las necesidades a cubrir por la cooperativa. Los mejores ejemplos en Chile son las cooperativas de vivienda abiertas y principales cooperativas de ahorro y crédito. 
- Según su carácter de importancia económica: Estas, están señaladas en el artículo 109 de la Ley de Cooperativas como todas aquellas entidades cuyos activos sean iguales o superiores a 50.000 Unidades de Fomento o que tengan más de 500 socios. Como ejemplos tenemos la totalidad de las cooperativas de ahorro y crédito y las cooperativas abiertas de vivienda. Hoy existen 165 entidades de este tipo, de las cuales $30 \%$ corresponden a entidades de ahorro y crédito (LEY de COOPERATIVAS, 2007).

Para efectos de este trabajo se privilegió como principal criterio el sectorial, presentando los datos debidamente actualizados al 2013 en la tabla 1.

Tabla 1. $N^{o}$ de Cooperativas y de Socios agregados por Sector de Actividad

\begin{tabular}{|c|cc|}
\hline TIPO & \multicolumn{2}{|c|}{ ACTIVAS Y VIGENTES } \\
\hline I. SECTOR COOPERATIVO & $\mathbf{1 3 2 4}$ & \\
\hline II.1 COOPERATIVAS DE PRODUCCIÓN & 440 & 135 \\
\hline III. 1.1. Cooperativas Agrícolas & & 236 \\
\hline III. 1.2. Cooperativas Campesinas & 635 \\
\hline III. 1.3. Cooperativas Pesqueras y Mineras & & 26 \\
\hline II.2 COOPERATIVAS DE SERVICIOS & & 45 \\
\hline III. 2.1. Cooperativas de Consumo & & 158 \\
\hline III. 2.2. Cooperativas Ahorro y Crédito & & 23 \\
\hline III. 2.3. Cooperativas Vivienda Abierta & & 220 \\
\hline III. 2.4. Cooperativas Vivienda Cerrada & \\
\hline III. 2.6. Cooperativas de Agua Potable & 230 \\
\hline III. 2.7. Cooperativas Eléctricas & 19 \\
\hline III. 2.8. Otros Servicios & \\
\hline II.3 COOPERATIVAS DE TRABAJO & & \\
\hline II.4. FEDERACIONES Y CONFEDERACIONES & & \\
\hline
\end{tabular}

Fuente: Construcción propia, basado en datos del DECOOP (2014)

El número de cooperativas vigentes al 31 de diciembre del 2013, registradas en el Departamento de Cooperativas del Ministerio de Economía ${ }^{4}$ es de 2.572. De estas, 1.324 se encuentran vigentes y activas. Su distribución por regiones se muestra en la Figura 4:

\footnotetext{
${ }^{4}$ Departamento de Cooperativas. Balance de Gestión 2010-2013. Enero 2014.
} 
Figura 4.Cooperativas Vigentes y Activas por Región

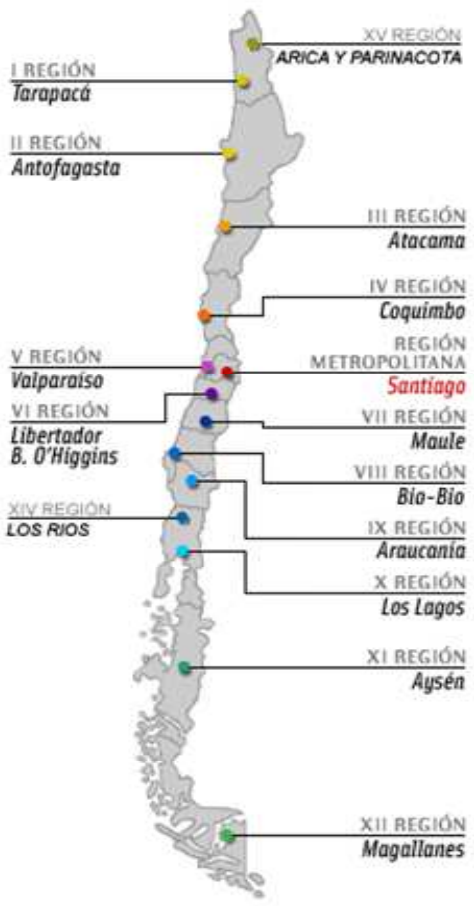

\begin{tabular}{|c|l|r|r|}
\hline & \multicolumn{1}{|c|}{ Región } & N ${ }^{\circ}$ Cooperativas \\
\hline XV & Región de Arica y Parinacota & 15 \\
\hline I & Región de Tarapacá & 24 \\
\hline II & Región de Antofagasta & 7 \\
\hline III & Región de Atacama & 15 \\
\hline IV & Región de Coquimbo & 70 \\
\hline V & Región de Valparaíso & 176 \\
\hline R.M. & Región Metropolitana & 341 \\
\hline VI & Región del Libertador B. O'Higgins & 91 \\
\hline VII & Región del Maule & 92 \\
\hline VIII & Región del Biobío & 130 \\
\hline IX & Región de Araucanía & 141 \\
\hline XIV & Región de Los Ríos & 37 \\
\hline X & Región de Los Lagos & 144 \\
\hline XI & Región de Aysén & 26 \\
\hline XII & Región de Magallanes & 15 \\
\hline Total Cooperativas & 1.324 \\
\hline & & \\
\hline
\end{tabular}

Fuente: DECOOP (2014)

\section{Niveles EstruCturALES - DESPLIEGUE DE COMPLEJIDAD DEL SCCH}

Posterior a la adopción de criterios de clasificación, y en base a los datos proporcionados por el Departamento de Cooperativas ${ }^{5}$, se definió como variable de representación y distribución de la complejidad del SCCh el número de cooperativas.

Siguiendo tales criterios se construyó la estructura recursiva para el cooperativismo chileno, representado en la figura 5. En la ilustración se configura una cascada de 4 niveles de complejidad, que comienza con el SCCh (Nivel 0). Luego, le siguen los sub-sectores (Nivel 1), integrados por producción, servicios y trabajo. A continuación, continúa en los sub-subsectores (Nivel 2), teniendo en cuenta que no existe simetría y que algunos sub-sectores tienen un nivel de desagregación mayor, cual es el caso de las cooperativa de servicio. Fimalmente, la recursividad estructural se expande hasta las cooperativas de base como último nivel recursivo.

${ }^{5}$ Cabe señalar que existe un registro de cooperativas en el sitio web del Departamento de Cooperativas del Ministerio de Economía www.decoop.cl. Sin embargo, la información aquí utilizada proviene de un informe oficial: Balance de Gestión Departamento de Cooperativas 2010- 2014, aportado por dicha repartición y no del sitio web, debido a que éste presenta algunos errores en sus datos, lo cual se espera sea corregido durante estos próximos meses. 
Figura 5. Despliegue de Complejidad del SCCh. según $\mathrm{N}^{o}$ de Cooperativas

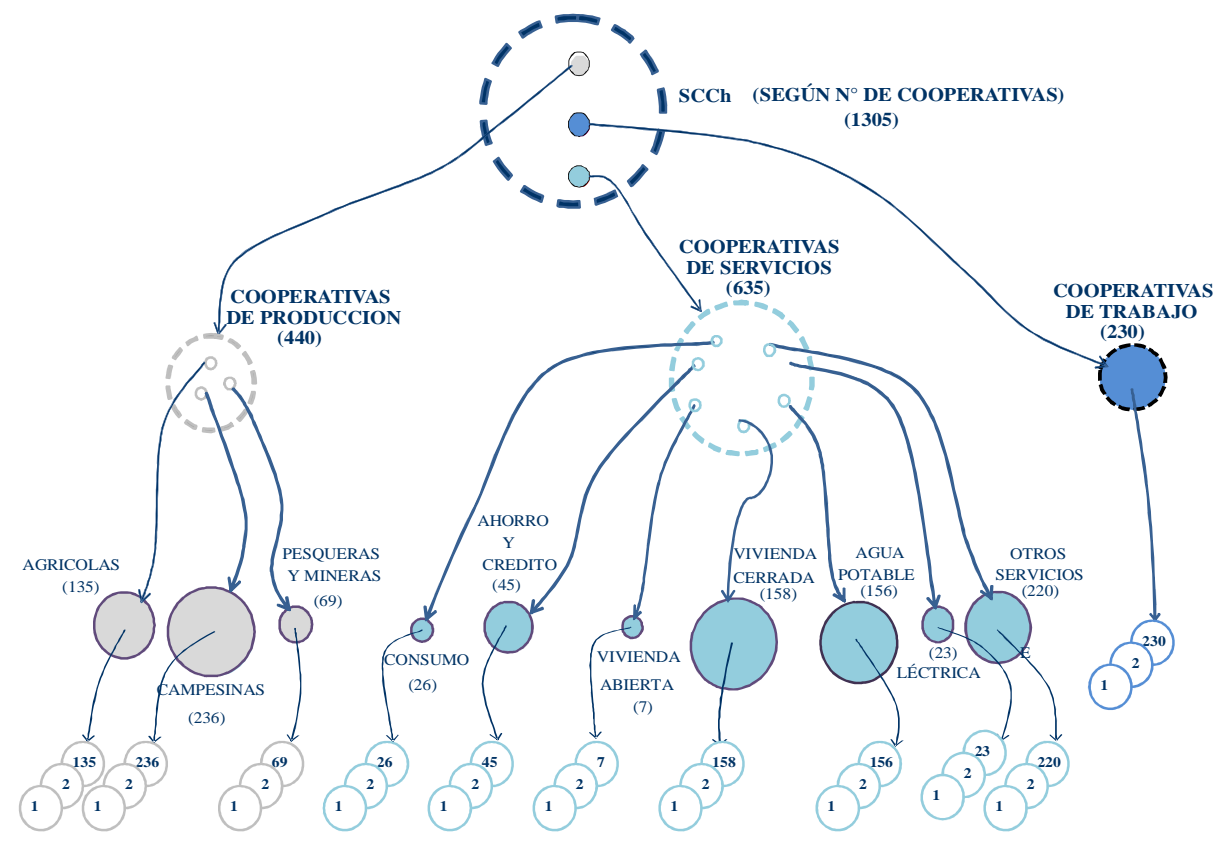

Fuente: Elaboración Propia

En la figura 5, los círculos representan a las actividades primarias de la organización y sus tamaños son proporcionales al número de cooperativas que integran cada actividad primaria. De ese modo, es posible visualizar el Sector Cooperativo como un todo y la forma en que se va desplegando su complejidad hacia sus subsistemas, luego a los sub-sub-sistemas y así sucesivamente. Estas figuras no muestran jerarquía, sino que un despliegue sucesivo de los subsistemas viables inmersos -en este caso desplegados- en los sistemas viables a los que pertenecen.

Algunos tipos de cooperativas, específicamente las federativas fueron excluidas de este análisis, ya que a nuestro juicio ellas, cibernéticamente hablando, no son actividades primarias $-\mathrm{o}$ productivas- en tanto no son viables, es decir, no son generadoras (productoras) de la organización. Complementariamente a la labor de las actividades primarias, tales entidades tienen una labor de apoyo a las sinergias operativas, estratégicas y normativas del sector, por lo cual deben ser abordadas junto a los mecanismos de centralización/ descentralización, regulación y control del sistema.

En lo relativo a los criterios de importancia económica y de abierta-cerrada, estos tendrán significado organizacional como propiedades de las cooperativas de base. 
Analizando la figura, y considerando la información secundaria recogida (DECOOP, 2014 y el Directorio de Cooperativas del DECOOP), podemos observar que:

En cuanto a tamaño: El sector más grande, por el número de cooperativas, corresponde a los servicios. Existen sectores o subsectores que destacan por el número de cooperativas con que cuentan. Ellos son: las cooperativas campesinas, de trabajo, vivienda cerrada, agrícolas y de agua potable, superando las 100 cooperativas.

Con respecto a número de socios: Es posible inferir los sectores o subsectores que tienen un importante presencia en el número de socios: consumo (con 129.824) y ahorro y crédito (con 1.238.370 socios).

En cuanto a concentración de socios (socios/cooperativas): Si profundizamos un poco más en la comparación de datos, podemos ver que algunos sub- sub-sectores poseen una alta concentración de socios por cooperativas. Entre estos se encuentran: consumo, ahorro y crédito, vivienda abierta, y las cooperativas eléctricas, superando todas ellas los 4000 socios en promedio por cooperativa. Simultáneamente llama la atención la baja cantidad de socios /cooperativa con que cuentan los su-sub- sectores de cooperativas campesinas, pesqueras y mineras, y las de trabajo, las cuales no superan los 10 socios promedio/cooperativa.

En Estabilidad y Desarrollo: Algunos sub-sub-sectores cooperativos nacen en períodos muy específicos del desarrollo económico nacional, y luego de la crisis sufrida por el sector en la década de los '80 se mantienen con una cierta inercia, cual es el caso de las cooperativas agrícolas en general. En otros segmentos, en tanto, es visible que luego del periodo de crisis en que fueron perseguidas y golpeadas por la dictadura, comienzan a tomar fuerza renovadora en la actualidad, como ha ocurrido con las cooperativas campesinas, de transporte y las de producción y trabajo. Por otra parte también existen entidades que sin llegar a desarrollarse mantienen su funcionamiento estable, como por ejemplo las cooperativas de Veraneo y de Salud (ambas de servicios). Algunos casos especiales a destacar son las de Vivienda Abierta y de Ahorro y Crédito, que aún en tiempos difíciles tuvieron la capacidad de reconvertirse en organizaciones competitivas.

Con respecto a la sobrevivencia: Para dar cuenta de la sobrevivencia, medimos el porcentaje de entidades vigentes y activas en relación con el número total de cooperativas creadas. De esta manera, constatamos un alto porcentajes de sobrevivencia entre las cooperativas agrícolas pisqueras y vitivinícolas, como también las de salud, con promedios sobre el $60 \%$. 
Mayor estabilidad aún alcanzan las cooperativas de agua potable, con una sobrevivencia del $81 \%$ de las 190 entidades creadas, y las cooperativas eléctricas con un 85\%. Como contraste, otros subsectores se muestran altamente inestables, entre ellos algunas subcategorías de agrícolas (hortícola y frutícola, lechera y avícola), con un promedio inferior al 30\%; lo cual es aún peor en las cooperativas mineras y de consumo, con porcentajes del $4 \%$ y $9 \%$ de supervivencia respectivamente.

\subsection{Centralización - descentralización}

En el punto anterior se definieron las actividades primarias del SCCh, y se estudió cómo se despliega su complejidad considerando como variable principal el (número de cooperativas).

En este punto, se pretende analizar la capacidad funcional del sector; entendida como el grado de centralización y descentralización que alcancen las políticas sectoriales. El correcto funcionamiento de la centralización-descentralización sectorial, es de responsabilidad compartida, tanto por las organizaciones representantes del mundo cooperativo (uniones, federaciones y confederaciones), como por los organismos reguladores gubernamentales

Del análisis de la información recolectada en relación a la organización sectorial, notamos una alta fragmentación, lo que debilita la capacidad representativa del SCCh como un todo sinérgico. Algunos sub-sub- sectores del mundo cooperativo chileno han hecho un esfuerzo para organizar su sector, como son los ejemplos de las cooperativas campesinas, agrupadas en CAMPOCOOP, y las de Ahorro y Crédito agrupadas en FECRECOOP. Estos ejemplos desgraciadamente no son replicados por el resto de los sub-sectores.

Barriendo la recursividad estructural, partiendo del nivel más bajo de complejidad (las cooperativas de base), podemos encontrar algunos pocos niveles de agregación voluntaria en entidades de segundo grado (como uniones y federaciones), y de tercer grado (en confederaciones). En resumen:

- Existen 15 federaciones activas de las 44 que se han formado. Entre ellas destaca FECRECOOP, que representa a uno de los subsectores más dinámicos del último tiempo: el de Ahorro y Crédito.

- Existen solo 2 confederaciones de las cuatro que se han creado: una que aglutina a las Federaciones Campesinas, y se llama CAMPOCOOP; y la otra, CONFECOOP, 
máximo líder "gremial" del cooperativismo Chileno.

- De las uniones, se encuentra activa 1 de 12 creadas.

- De las 6 cooperativas de servicios auxiliares que se han creado, sólo dos permanecen activas, a saber: el Instituto de formación cooperativo ICECOOP $^{6}$ y la auditora AUDICOOP. La primera de ellas, dedicada a la formación y consultoría en temas cooperativos y la otra corresponde al único instrumento creado al interior del movimiento cooperativo que se desempeña en actividades de autocontrol y auditoría del sector cooperativo.

Las organizaciones de segundo y tercer grado son vitales para tejer un movimiento cooperativo viable, por lo cual debieran actuar como pilares para la articulación del desarrollo del sector, participando activamente en la generación de estrategias de desarrollo. Por ello, sostenemos que las cooperativas de base deberían invertir esfuerzos en la generación de estructuras que apoyen la representación política, la investigación y desarrollo y otras sinergias imprescindibles para su desarrollo. Esto debe ser diseñado e implementado en todos los niveles recursivos de la estructura sectorial.

La actual arquitectura federativa tiene -desde nuestro punto de vista- falencias sustantivas en su diseño legal que le impiden potenciar las sinergias organizacionales. Si bien las figuras jurídicas posibles para estas superestructuras se encuentran definidas, no es clara la manera en que pueden ordenarse en una lógica orgánica. Se advierte, además, la ausencia de un ente representativo del movimiento en su conjunto.

Desde el ámbito gubernamental, la preocupación por el SCCh, recae en el Departamento de Cooperativas del MINECON, que constituye el principal organismos regulador del Sector. Su rol está establecido por la Ley General de Cooperativas en su capítulo IV, enfocándose en el "fomento del sector cooperativo mediante la promoción de programas destinados al desarrollo de la gestión y la capacidad empresarial, la dictación de normas que contribuyan al perfeccionamiento del funcionamiento de las cooperativas, el registro de cooperativas vigentes y la supervisión y fiscalización de las mismas" (DECOOP, 2014). Hoy el DECOOP está en un proceso de reestructuración, el que podría potenciar las

\footnotetext{
${ }^{6}$ ICECOOP promueve y ejecuta actividades de formación de recursos humanos y realiza consultorías a cooperativas, empresas asociativas y pequeños empresarios. Dicta cursos de capacitación, asistencia técnica y asesorías y formación de técnicos en administración.
} 
capacidades de fomento desde el Estado, para con el Sector.

En síntesis, las prácticas de apoyo sectorial necesarias para la viabilidad cooperativa, tanto por parte del Estado como del mundo cooperativo, que debieran permitir: el acceso de las cooperativas a las fuentes de financiamiento, la planificación estratégica del sector, el fomento a la innovación tecnológica y a la creación de nuevas iniciativas cooperativas, se encuentran hoy debilitadas.

\subsection{Mecanismos reguladores}

En esta etapa se clausura el diagnóstico del sector analizándose los elementos destinados a lograr una administración eficiente y efectiva. Para ello, proponemos un modelamiento de la estructura organizacional siguiendo la lógica establecida por el MSV. Cabe señalar que tal modelamiento no pretende fijar la estructura final, sino ser una guía que posibilite orientaciones de cambio en la organización de cara al medio externo.

La Figura 6 ilustra el diagnóstico del SCCh desde la perspectiva del Modelo del Sistema Viable. El modelo construido e ilustrado en la figura, representa el nivel de recursión agregado para todo el SCCh.

Dada la fragmentación del Sector, la que debilita su viabilidad, no se justifica construir modelos diagnósticos de los sectores intermedios (sub-sectores y sub-sub-sectores), los que actualmente sólo son agregaciones virtuales lógicas de cooperativas según algún criterio determinado.

Al examinar la figura podemos diferenciar dos áreas de vital importancia para la viabilidad del SCCh: el área de IMPLEMENTACIONES, compuesta por las cooperativas de base que son el objeto de control y desarrollo del sistema, y el área de METASISTEMA, encargada de regular el sector para el cuidado de su viabilidad. 
Figura 6. MSV para el Sector Cooperativo Chileno

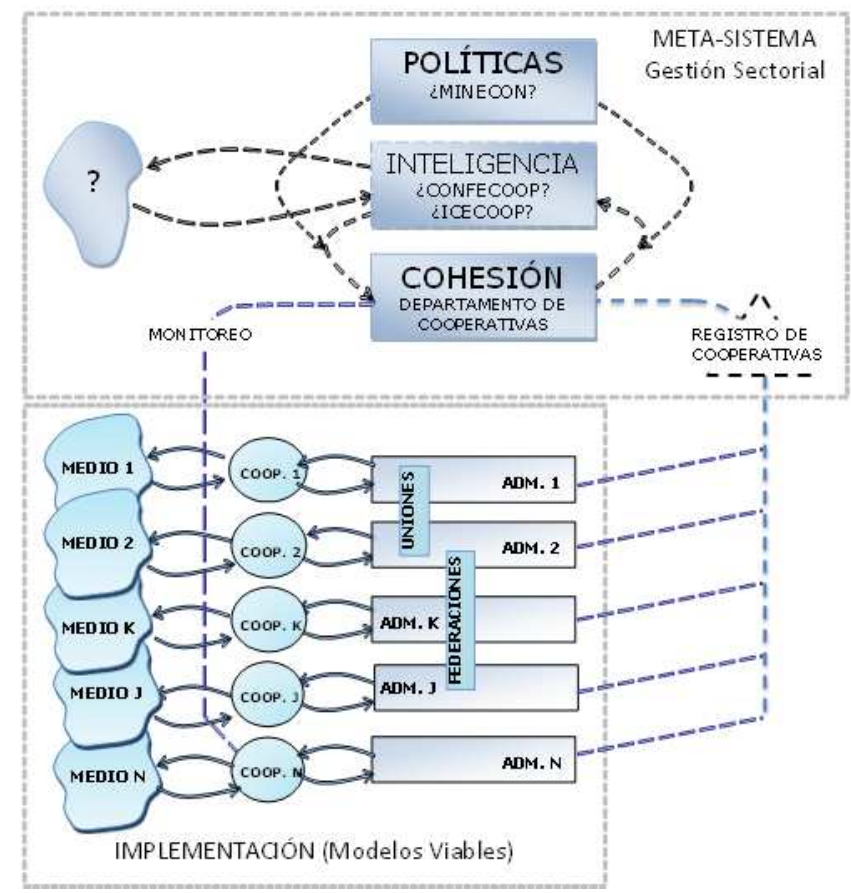

Fuente: Elaboración Propia

\section{ÁREA DE IMPLEMENTACIONES}

En la figura 6, las cooperativas de base están representadas como círculos, cada cual con su entorno relevante dibujado con línea discontinua, y con su administración vista como un rectángulo.

Sin entrar a discutir la viabilidad de las cooperativas de manera individual, podemos notar que en términos operacionales se observan interacciones débiles y en muchos casos inexistentes entre las cooperativas de base, aunque sus entornos económicos, geográficos y tecnológicos puedan estar relacionados. Al nivel de sus administraciones, en algunos casos existe cierta interacción mediante algunas superestructuras, como son las ya analizadas uniones y federaciones, donde algunos grupos han tomado cierta fuerza sectorial, tales como CAMPOCOOP y FECRECOOP.

\section{ÁREA DE METASISTEMA}

a) Diagnóstico para el mecanismo de cohesión organizacional (La relación de operación entre los Subsistemas 1,2 y 3 del MSV). 
Desde un punto de vista cibernético, controlar es cuidar la estabilidad y cohesión de las operaciones. En el caso del SCCh, su responsable está claramente identificado y es el Departamento de Cooperativas del MINECON.

El Departamento de Cooperativas, como forma de absorber la complejidad administrativa de las organizaciones mantiene un registro general de éstas, disponible en su

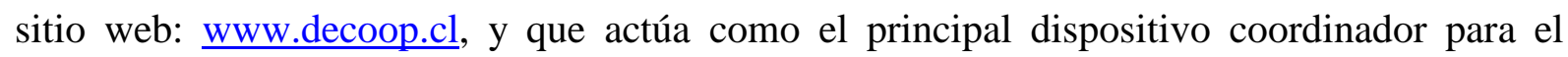
SCCh. Esta coordinación actualmente se encuentra en una interesante etapa de modernización y fortalecimiento de sus funciones, principalmente a través del Programa de Fortalecimiento de la Regulación y Supervisión del Sector Cooperativo Chileno.

b) Diagnóstico para el mecanismo de adaptación (La relación de operación entre los Subsistemas 3, 4 y 5 del MSV).

Al estudiar la identidad organizacional concluimos que el SCCh se encuentra fragmentado o más bien desorientado. Estructuralmente, eso tiene relación con el mecanismo de adaptación, que es el dispositivo estructural que da orientación y sentido a las organizaciones en la relación con su entorno relevante.

El mecanismo de adaptación se fundamenta en tres funciones vinculadas entre sí: la función de políticas, preocupada del cuidado por la identidad organizacional del sistema; la de inteligencia, encargada del futuro pesquisando las oportunidades y amenazas en el entorno; y la función de cohesión, que vela por el desarrollo de las fortalezas y la superación de las debilidades para el equilibrio operacional y la eficiencia de la organización.

Con respecto a la función de políticas, advertimos que se encuentra prácticamente abandonada, ya que el MINECON, responsable de entregar las grandes direcciones para el $\mathrm{SCCh}$, en la actualidad focaliza su interés en otras áreas. Un claro ejemplo de ello es el Proyecto de Ley que modificó la Ley General de Cooperativas, que demoró más de una década en su tramitación en el Congreso de la República sin que el ejecutivo le definiera especial urgencia.

Como función inteligencia, las instancias que deberían ser las encargadas de establecer la orientación estratégica del SCCh y conducir su modernización, coordinando los esfuerzos con todos los agentes sociales con vocación de economía social, son el Instituto Chileno de Educación Cooperativa -en adelante ICECOOP-, como Instituto de formación y de estudios 
cooperativos, y la CONFECOOP, como principal representante del Sector. Estos organismos llevan a cabo estudios y generan bibliografía, sin embargo, carecen de una actitud más proactiva que los transforme en coordinadores estratégicos. En la actualidad ellos se encuentran faltos de recursos, atrasados tecnológicamente y coartados por la actual legislación.

Sumado a las falencias propias de las funciones de políticas, inteligencia y cohesión sectoriales, se encuentra la falta de solidez estratégica entre estos componentes. Creemos que una vía para la superación de esta realidad es la generación de un plan estratégico o lineamientos estratégicos del sector por parte del conjunto de actores relevantes involucrados. Múltiples mecanismos se pueden utilizar para estos fines: seminarios sectoriales de discusión y acuerdos, comisiones de alto nivel que establezcan las líneas estratégicas para el sector, o también la generación de un equipo técnico que establezca un plan base para discutirlo e implementarlo sectorialmente.

Como podemos notar, desde un contexto de viabilidad, la estructura actual del SCCh no permite un accionar saludable. Muchos integrantes del entorno cooperativo, tenían puestos los ojos en que la nueva ley sería la solución para el sector. Los hechos están mostrando que no; la ley permita algunos alivios al estrés que vive el sector en la actualidad, sin embargo, la estructura organizacional del SCCh requiere modificaciones sustanciales que fortalezcan su orgánica, especialmente en lo que dice relación con la adaptación y el desarrollo.

\section{ORIENTACIONES PARA EL DESARROLLO ORGANIZACIONAL DEL SCCh}

Establecimos la implementación de ciertas pautas de actuación en el corto plazo, denominadas "acciones organizacionales" en cuatro ámbitos específicos para la viabilidad sectorial, a saber: en las políticas, en el ámbito de la identidad, la inteligencia, y la cohesión organizacional. El propósito de plantear acciones organizacionales es darle clausura sistémica a las orientaciones estratégicas, proveyendo de la capacidad funcional necesaria para conseguir una adaptación dinámica del Sector. Entre estas acciones enfatizamos:

- Identidad. La implementación de acciones concretas para fortalecer y visibilizar al sector corresponde tanto a las cooperativas de base, como a los órganos colegiados y al Departamento de Cooperativas. 
Respecto a las cooperativas de base, si bien es difícil -y poco asertivo- establecer acciones estandarizadas, ya que ello dependerá de las características económicas y sociodemográficas de cada una, en términos generales se considera pertinente masificar entre el mundo empresarial (micro.-pequeña y gran empresa) los beneficios del modelo cooperativo en términos organizacionales y económicos. En cuanto a los órganos de representación, sus actividades promotoras de identidad se relacionan con la organización de actividades de difusión por una parte, de capacitación por otra, de manera autónoma mancomunada con sus cooperativas afiliadas y con el Departamento de Cooperativas.

- Inteligencia. Al estudiar el SCCh advertimos el grado de desarticulación que caracteriza su actual estructura federativa, lo que constituye un factor inhibitorio para canalizar cualquier estrategia de desarrollo debido a su baja capacidad funcional. Al respecto, se propone la realización de análisis evaluativos y propositivos del Sector sistemáticamente. Estas actividades deben ser asumidas tanto por el macro- organismo de representación, como por las entidades de servicios auxiliares, a saber:

La COONFECOOP, debería ser el articulador de este grupo y le cabe la representación frente al mundo político. Para ello debe relacionarse con el MINECON, los partidos políticos, parlamentarios y organizaciones internacionales -como la ACI y otras-.

El ICECOOP, tendrá un rol coordinador y aglutinador de las instancias de formación, educación e investigación; para ello debe mantener continuas relaciones con centros de formación superior, medio y básico, para abrir la educación y la investigación cooperativa a la ciudadanía. Debe buscar programas de difusión cooperativa, mediante libros, revistas y folletos. Además, en conjunto con las Universidades desarrollar programas como: seminarios, cursos formativos y concursos acerca de emprendizaje cooperativo.

El Instituto de Auditoría Cooperativo -AUDICOOP- cumplirá el rol técnico-económico, y debe proveer capacidad de innovación. Para ello, deberá administrar una cartera de consultores que ofrezcan servicios de desarrollo empresarial cooperativo.

Algunos aspectos importantes de tener en cuenta en el entorno cooperativo son: ámbito tecnológico, entendido como potenciamiento de la innovación y de la capacidad emprendedora; ámbito financiamiento, ocupado de la búsqueda de recursos y/o oportunidades de negocios para el Sector; ámbito legislativo, relativo a las necesidades normativas y sus posibles adecuaciones; abordaje de nuevas temáticas de creciente 
relevancia social, tales como medio ambiente y recursos naturales, reivindicaciones étnicas e igualdad de oportunidades para grupos vulnerables.

- Cohesión. Apoyar la función de cohesión que le corresponde al Departamento de Cooperativas. Esta Oficina Ministerial debe mantener la cohesión para el SCCh y procurar las sinergias sectoriales.

Para hacerlo, el DECOOP cuenta con una Unidad Legal y de Registro y Archivos, que debe potenciarse tecnológicamente y mantener actualizada y normalizada la información existente. Cabe señalar que estas acciones permitirán a la vez alimentar a la investigación aplicada sobre el Sector.

Además de las labores de coordinación, el Departamento lleva a cabo auditorías que verifiquen la información reportada por los canales coordinadores. Respecto a ello, cabe señalar que a la fecha se están desarrollando estudios en el área de supervisión, que debieran decantar en modelos de fiscalización más simples y transparentes que faciliten tanto la gestión del DECOOP como de las propias cooperativas auditadas.

- Políticas. En el ámbito de las políticas se advierte la necesidad de mayor presencia del Ministerio de Economía en dos ámbitos: en el reconocimiento de la responsabilidad que le cabe en generar políticas fortalecedoras del SCCh; y en el otorgarle mayor visibilidad al cooperativismo y a la economía social como una alternativa válida y un factor estratégico para el desarrollo económico

Una última acción organizacional propuesta en el ámbito de las políticas dice relación con la creación de una instancia coordinadora de estrategias, que denominamos "Consejo Superior de Cooperativas". Esta propuesta surge tras el análisis general del sector, y de la constatación de que la inexistencia de un órgano interinstitucional que se encargue de que las orientaciones de futuro que se construyan sinérgicamente desde la política pública y desde las necesidades de las cooperativas de base. A partir de estas verificaciones, si bien valoramos positivamente los esfuerzos del DECOOP a través del Programa de Fortalecimiento de la Regulación y Supervisión del Sector Cooperativo Chileno, hacemos ver la necesidad de una instancia legitimada que dinamice las actividades del Sector como un proceso, y no como ejercicios aislados y fragmentados. 
Se propone que esta instancia público-privada esté constituida por los principales actores del movimiento cooperativo, tales como: un representante ministerial; los Directores de CONFECOOP, ICECOOP y AUDICOOP; el Director del Departamento de Cooperativas; representantes de las cooperativas de base por subsectores; representantes del poder legislativo. En lo jerárquico, esta unidad deberá ser autónoma con respecto al Poder Ejecutivo, aunque respetuosa de la Ley y de las políticas sectoriales dictadas por el MINECON. Se plantea una estructura liviana, cuyo financiamiento provenga de las entidades participantes, al menos hasta que probada su eficacia, se adscriba a la norma jurídica.

\section{CONCLUSIONES}

\subsection{Comentarios sobre la viabilidad del sector}

En relación a la caracterización del objeto de estudio, se pudo definir una sintomatología del SCCh en que destacaron factores tales como: un conflicto valorativo entre competitividad y cooperación al interior del mundo cooperativo; la indiferencia del mundo político y legislativo con el sector; el nacimiento del nuevo socio cooperativo -como aquel que solo busca ser servido por el sistema, sin comprometerse-, y el individualismo institucional -comportamiento competitivo entre las cooperativas-.

Del estudio del Sector Cooperativo Chileno -SCCh-, se concluyó con un modelo diagnóstico de su viabilidad. Nos muestra una organización con: una identidad débil -se nota un sector desconcertado-, alta fragmentación, heterogeneidad estructural -en términos de complejidad-, una regulación pobre, poca capacidad de adaptación y ausencia de políticas claras por parte del Gobierno.

Siguiendo con lo anterior, consideramos que uno de los aportes más relevantes del enfoque teórico y metodológico de la investigación realizada tiene que ver con que observa desde el sustento de la viabilidad del sistema. Esto supera las instancias que se enfocan en lo descriptivo analítico que no solamente diagnostican dificultades y oportunidades en torno a las debilidades y fortalezas de la entidad bajo estudio. Sino que a partir de un análisis de los diversos discursos - formales y subjetivos - ofrece una propuesta estratégica concreta en torno a los pasos a seguir por parte del sector. 


\subsection{Caracterización de la situación problema}

En relación a la caracterización del sector se pudo identificar 3 ámbitos que orientan la propuesta de comentarios, soluciones y recomendaciones. Estos son; estructura, regulaciones e intercooperación.

\section{Estructura}

- Desde un contexto de viabilidad, se pudo notar que la estructura actual del SCCh no permite un operar saludable. Al respecto se esperaba que la nueva ley fuera una solución, sin embargo ésta no incorpora modificaciones profundas que fortalezcan su orgánica, en especial lo que refiere a adaptación y desarrollo

- Potenciar el liderazgo del sector, tanto hacia las cooperativas de base, como en las relaciones con el mundo político, legislativo y gubernamental.

Regulaciones

- Potenciar las capacidades de gestión de las empresas cooperativas mediante la incorporación de instrumentos y prácticas de innovación tecnológica, planificación estratégica, comunicación con el medio, y sistemas de administración eficientes.

- Potenciar las capacidades de gestión de las empresas cooperativas mediante la incorporación de instrumentos y prácticas de innovación tecnológica, planificación estratégica, comunicación con el medio, y sistemas de administración eficientes.

Intercooperación

- Potenciar la educación cooperativa, en especial en un sentido valórico, técnico y de intercooperación. En este sentido es importante trabajar, formativa y culturalmente, el compromiso y participación de los socios cooperativistas, tratando de disminuir el fenómeno del nuevo socio. Del mismo modo, estudiar y promover los mecanismos de participación económica y en la gestión de los trabajadores asalariados del mundo cooperativo de servicios.

- Fomentar la intercooperación como una práctica fundamental para la viabilidad cooperativa. Esto, con el fin de recomponer el Movimiento Cooperativo en Chile-, y de generar sinergias de negocios 
- Generar redes de influencia dirigidas a ganar posiciones frente al mundo financiero y a los organismos de fomento estatal. Del mismo modo, se deben idear lazos importantes con las Cooperativas de Crédito, especialmente con las más poderosas para avanzar en una estrategia de Banco(s) Cooperativo(s), que puedan apoyar desde una mirada de Economía Social, el desarrollo del SCCh.

\subsection{Diagnóstico organizaciones}

Al revisar el sector cooperativo y las organizaciones que lo conforman, se puede observar que existen ciertos elementos que constituyen un desafío para el desarrollo del sector.

En relación a prácticas cotidianas, se observa que las prácticas empresariales se centran en la satisfacción de un grupo dentro del sector, en vez de orientarse a permitir eficiencia y efectividad para extenderse a un mercado competitivo.

En general se reconoce que el SCCh se presenta como uno donde se puede reconocer su funcionamiento, aun cuando se presenta como sumamente heterogéneo. Por lo mismo, cualquier incidencia o cambio orientado hacia el sector debe considerar la complejidad del mismo.

Considerando lo anterior se puedo observar también que es sector que encuentra organizacionalmente fragmentado y que esta actual estructura no permite un accionar saludable.

\subsection{Aportes y consideraciones teórico- metodológicas}

Para estudiar el SCCh -un sistema de actividad humana que por su heterogeneidad y el estado de fragmentación en que se encuentra, posee una gran complejidad empresarial y cultural- fue necesario abrir la mente a la gran variedad de percepciones que los distintos observadores tienen al respecto, para, a partir de este mapa de ideas, interpretar el espíritu de convergencia que da sentido al sistema como una totalidad.

Al respecto el enfoque teórico metodológico de la investigación resulta pertinente y compatible con el caso de estudio; una mirada holística y no reduccionista permite abordar un sector económico que presenta múltiples realidades y problemáticas. Junto con lo anterior, este enfoque permite ir más allá del estudio fenomenológico; donde la caracterización y 
descripción son un aporte pero no necesariamente ofrecen una solución contundente para generar cambios y superar las debilidades.

Así, se buscó estudiar el Sector Cooperativo Chileno como una organización de actividad humana, viva, que se ve enfrentada a cambios y realidades socio históricas que son multicausales; y al mismo tiempo presenta una complejidad interna que debe ser abordada desde una mirada holista.

También, fue adecuado incluir elementos de la cibernética organizacional, tanto en lo relativo a contar con un modelo poderoso que diera las referencias para el modelamiento, como por el método de estudio VIPLAN, el que guió la secuencia de pasos para el estudio de la viabilidad organizacional.

Esta proposición teórico-metodológica, permitió dejar expuesto un mecanismo de estudio para el SCCh, el que queda a disposición de los estudiosos del cooperativismo, tanto para replantear el diagnóstico, como para intervenir las demás recursiones, llegando incluso si fuese deseable, hasta las propias cooperativas de base.

El uso del Modelo del Sistema Viable -MSV- como referencia estructural y el VIPLAN como método de estudio, aplicado a un Sector económico, representó un desafío metodológico, ya que las orgánicas sectoriales suelen ser poco estructuradas y los límites de participación de sus actores bastante difusos. Sin embargo, y a pesar de las dificultades, quedó demostrado que la metodología propuesta puede ser una importante ayuda para estudiar y estructurar organizaciones de carácter sectorial y federativas.

Al respecto se puede decir que, desde la perspectiva teórica metodológica utilizada, los principales aportes del estudio son:

A. Presentamos una metodología novedosa para el estudio de organizaciones complejas, la que permite abordar una amplia gama de fenómenos organizacionales, ya que la única exigencia requerida es que persigan ser viables.

B. Entregamos un modelo diagnóstico del SCCh, el que además de darles estructura -desde una mirada global- denuncia sus debilidades actuales.

C. Proponemos un modelo que permitiría recomponer, con un sentido estratégico el SCCh. 
Por otro lado, este trabajo, entendido como un proceso que nos posiciona en el trabajo acerca de la economía social en general y del cooperativismo chileno en particular, nos abre una serie de opciones y desafíos para la investigación futura. Algunas de las líneas de investigación que se derivan del trabajo son:

I. "Estudio de la viabilidad de los subsectores cooperativos chilenos". Esto, como la posibilidad metodológica de efectuar el mismo procedimiento diagnóstico que realizamos, abarcando los subsectores cooperativos.

II. "Emprendizaje cooperativo". Esta línea de trabajo, se orienta a investigar en un sentido de investigación-acción, prácticas empresariales novedosas y efectivas en un contexto cooperativista sustentado desde la base social.

\section{BIBLIOGRAFÍA}

BIBLIOTECA DEL CONGRESO NACIONAL DE CHILE (2002) Modifica la Ley General de Cooperativas. Biblioteca Electrónica del Congreso Nacional Diario Oficial 04 de noviembre, 2002. Disponible en Internet: http://www.bcn.cl.

BEER S. (1985) Diagnosing the system for organizations. Décima edición. Chichester: Wiley. 152 p. ISBN: 0471906751.

CHECKLAND, P. y POULTER, J. (2009) Soft Systems Methodology: Método radical para integrar actividades organizativas. Milrazones. p. 192. ISBN: 8493755218.

DECOOP. (2014) Balance de Gestión Departamento de Coooperativas 2010-2014. Unidad de capacitación, estudio y difusión, Departamento de Cooperativas. [Publicación Interna].

DEL CAMPO, P. y RADRIGÁN, M. (1998) El Sector Cooperativo Chileno: Tradición, Experiencias y Proyecciones. CONFECOOP - CCA, Santiago de Chile.

ESPEJO, R., SCHUHMAN, W., SCHWANINGER, M y BILELLO, U. (1996) Organizational transformation and learning. $1^{\circ}$ Edición. Londres. Editorial Wiley. p. 364. ISBN: 0471961825.

ESPEJO, R. BOWLING, D. y HOOVERSADT, P. (1999) The viable system model and the Viplan Software. Kybernetes: The international journal of systems, cybernetics and management science. Vol. 28, $\mathrm{N}^{\mathrm{0}}$ 6/7, pp. 661 - 678. Disponible en Internet: http://www.emeraldinsight.com/journals.htm?articleid=875824. (ISSN: 0368-492X) DOI: $10.1108 / 03684929910282944$. 
ESPEJO R. (2003) The Viable System model: a briefing about organisational structure. SYNCHO Limited. [en línea] Syncho Research \& Dissemination on Managing Complexity. [23 de enero de 2012] Disponible en Internet: http://www.syncho.com/pages/pdf/INTRODUCTION\%20TO\%20THE\%20\%SYSTEM\% 20MODELS3.pdf.

ESPEJO, R. y REYES, A. (2011) Organizational System: Managing Complexity with the Viable System Model. $1^{\mathrm{a}}$ edición. Londres. Springer Science \& Business Media. p. 276. ISBN: 3642191091.

ESPEJO, R. (2015) An Enterprise Complexity Model: Variety Engineering and Dynamic Capabilities. International Journal of Systems and Society, Vol. 2, № 1, pp 1-22. DOI: 10.4018/ijss.2015010101.

LÓPEZ GARAY, H. (1995): Proyecto de una plataforma de base para pensar sistémicamente el problema del desarrollo de América Latina. Sistémica' 94, Instituto Andino de Sistemas. pp. 265-294.

LÓPEZ GARAY, H. (1997): En busca de paradigmas socio políticos alternos para América Latina: una reflexión sistémica preliminar. I Coloquio Ibero-americano sobre Ciencia, Tecnología y Sociedad, Santiago de Chile. p. 20.

MINISTERIO DE ECONOMÍA (2006) Panorama general del sector cooperativo chileno.

Departamento de Cooperativas. Unidad de Fomento, Capacitación y Estudios. [Comunicación privada].

MINISTERIO DE ECONOMÍA, FOMENTO Y RECONSTRUCCIÓN (2007) Ley General de Cooperativas. Decreto Supremo 502 de 1978 fija texto de la Ley General de Cooperativas.[en línea] Ley 20190 del 05.06.2007. Biblioteca Electrónica del Congreso Nacional. Disponible en Internet: http://www.bcn.cl.

MEDINA, E. (2013) Revolucionarios Cibernéticos: Tecnología y Política en el Chile de Salvador Allende. LOM Ediciones. p. 358. ISBN: 9560004352.

MORGAN, G. (1999) Imagin-i-zación. Granica. p. 456. ISBN: 8475777686.

NARVARTE, P. (1995) Metodología sistémico-cibernética para la flexibilización de organizaciones públicas: el caso de una unidad académica en el contexto universitario chileno. SISTÉMICA’94, 2da. Conferencia Internacional de Trabajo del Instituto Andino de Sistemas (IAS). Primera Edición. Lima, Perú. Ediciones IAS. p. 296. ISBN: 8460527921. 
NARVARTE, P. (2002) Estudio de la Organización Cooperativa como un Sistema Viable: la Experiencia de Mondragón como una Referencia Estratégica para el Desarrollo del Sector en Chile. Tesis doctoral, Mondragón Unibertsitatea.

NARVARTE, P., IBARRA, E. y BAEZA, K. (2002) Metodología para el desarrollo de proyectos asociativos. En MARTÍ, J.P., PÉREZ DE URALDE , J.M.(coord.) La Autonomía y la Autogestión en las Cooperativas. Herramientas y metodologías para su desarrollo. Divina Pastora Fundación. pp. 34-45. ISBN: 84-693-6066-8.

NARVARTE, P. y RODES, D. (2004) Expresión de la situación problema para el sector cooperativo chileno: una aproximación sistémica. Revista de Cooperación Internacional de la ACI (Asociación Cooperativa Internacional).Vol. 37, № 1- 2004, pp. 7-22.

NARVARTE, P. (2006) La Experiencia Cooperativa de Mondragón: estudio de su viabilidad organizacional en el contexto de Euskadi. CIRIEC-España, Revista de Economía Pública, Social y Cooperativa, $\mathrm{N}^{\mathrm{o}}$ 54, pp. 231-255.

NARVARTE, P., CASTILlO, C. y TORRES, N. (2006) Estudio de la Viabilidad Organizacional del Sector Cooperativo de Ahorro y Crédito en Chile. ler Coloquio Internacional de la Red RULESCOOP, Universidad de Bretagne Occidentale, Brest Francia, 2006. 14 pgs.

NARVARTE, P., ÁlvAREZ, S. y BAEZA, K. (2014) Reflexión Sistémica del Fenómeno Económico: Propuestas para entender la Crisis. En FAJARDO, G., SENET, M. J. (Coord.). Economía Social: Identidad, Desafíos y Estrategias. CIRIEC- España. pp. 141152. ISBN: 8494126017.

PEREZ, E., RADRIGÁN, M. y MARTINI, G. (2003) Situación actual del cooperativismo en Chile. Santiago, Chile: Pro-Asocia- Universidad de Chile, p. 79.

RODRÍGUEZ, R. (1994) La sistémica, los sistemas blandos y los sistemas de información. Primera Edición. Lima, Perú: Universidad del Pacífico. p. 159. ISBN: 8489293. 